\title{
Diarrheal disease risk in rural Bangladesh decreases as tubewell density increases: a zero- inflated and geographically weighted analysis
}

Margaret Carrel', Veronica Escamilla², Jane Messina², Sophia Giebultowicz², Jennifer Winston², Mohammad Yunus ${ }^{3}$, P Kim Streatfield ${ }^{3}$ and Michael Emch ${ }^{2 *}$

\begin{abstract}
Background: This study investigates the impact of tubewell user density on cholera and shigellosis events in Matlab, Bangladesh between 2002 and 2004. Household-level demographic, health, and water infrastructure data were incorporated into a local geographic information systems (GIS) database. Geographically-weighted regression (GWR) models were constructed to identify spatial variation of relationships across the study area. Zero-inflated negative binomial regression models were run to simultaneously measure the likelihood of increased magnitude of disease events and the likelihood of zero cholera or shigellosis events. The aim of this study was to examine the effect of tubewell density on both the occurrence of diarrheal disease and the magnitude of diarrheal disease incidence.
\end{abstract}

Results: In Matlab, households with greater tubewell density were more likely to report zero cholera or shigellosis events. Results for both cholera and shigellosis GWR models suggest that tubewell density effects are spatially stationary and the use of non-spatial statistical methods is appropriate.

Conclusions: Increasing the amount of drinking water available to households through increased density of tubewells contributed to lower reports of cholera and shigellosis events in rural Bangladesh. Our findings demonstrate the importance of tubewell installation and access to groundwater in reducing diarrheal disease events in the developing world.

\section{Background}

Matlab, Bangladesh is a rural area located approximately 50 kilometers southeast of the capital city, Dhaka. Diarrheal diseases are endemic in Matlab and across Bangladesh. This is due to a number of factors, including natural aquatic environments of diarrheal causative agents, high population density, low socioeconomic status, and limited access to clean water. The installation of tubewells is a primary method for decreasing diarrheal disease incidence, giving Bangladeshis an alternative to drinking contaminated surface water. These tubewells draw water several hundred feet from the underlying aquifer to the surface by hand pumps. A sanitary seal, typically of concrete, ideally prevents

\footnotetext{
* Correspondence: emch@email.unc.edu

${ }^{2}$ Department of Geography \& Carolina Population Center, University of North Carolina-Chapel Hill, Chapel Hill, NC, USA

Full list of author information is available at the end of the article
}

seepage of ground water into the tubewell. Despite their sealed nature, tubewells may still harbor microorganisms, such as fecal bacteria, as a result of their proximity to latrines or contaminated surface water bodies [1].

Despite such possible contamination of tubewell water, previous studies of diarrheal disease and drinking water interactions have suggested that it is not simply the use of alternative groundwater sources that provides protection against infection, but also the quantity and accessibility [2-7]. Put simply, the density of a population sharing a tubewell can lower the protective effect conferred by a water supply. Our study examines the potential impacts of tubewell access on two distinct types of diarrheal disease events among Matlab's residents between 2002 and 2004. In doing so, we address two problems commonly encountered and often overlooked in disease analysis: spatial variation in relationships and a small number of reported events.

\section{C) Biomed Central}


Geographically weighted regression is used to assess potential spatial variability in relationships across the study area and zero-inflated models to control for low disease counts. By utilizing both methods, we are able to ascertain the impact of tubewell access on cholera and shigellosis patterns without the confounding effects of spatial non-stationarity or rare event reporting.

Cholera and shigellosis are two of the most commonly experienced diarrheal diseases in Matlab. Cholera is a disease caused by ingesting a large number of Vibrio cholerae bacteria; the infective dose is 10,000 or greater. Shigellosis is caused by ingesting a much smaller dose of Shigella bacteria; sometimes as few as ten bacteria are necessary to cause an infection. The primary symptom of cholera is watery diarrhea, while the primary symptom of shigellosis is bloody diarrhea (dysentery). Cholera bacteria naturally occur in the brackish waters of Bangladesh, while Shigella bacteria are linked to human waste contamination of water supplies. We hypothesized that, despite these described differences between the two diseases, tubewell user density would be predictive of diarrheal events for both.

\section{Methods \\ DATA}

Since 1966, the International Centre for Diarrhoeal Disease Research, Bangladesh (ICDDR,B) has conducted continuous demographic surveillance system (DSS) of Matlab's residents. Each resident is assigned a unique identification number which is used to track their birth, place of residence, migration, health events, marriage, and eventually, death. The 225,000 residents of Matlab live in a network of baris, or patrilineally related household clusters, which are then further grouped within 142 villages (Figure 1). Community health workers trained by ICDDR,B visit each bari in Matlab once a month and record a variety of demographic and health events which are linked via the identification number to both individual resident's history, the household and bari history, and village history.

ICDDR,B operates a hospital in southwest Matlab that specializes in high-quality treatment of diarrheal diseases, respiratory infections, and maternal and child health. Treatment at the ICDDR,B is free for residents of Matlab, and free transportation to the hospital is available for residents who cannot afford travel. The laboratory diagnoses and treatment outcomes of patients are linked to their demographic and health records via the resident's identification number.

The health and demographic surveillance data collected by ICDDR,B is linked to the spatial locations of residence via a Geographic Information System (GIS). The latitude and longitude coordinates collected at the center of each bari are geocoded in the GIS, as are the

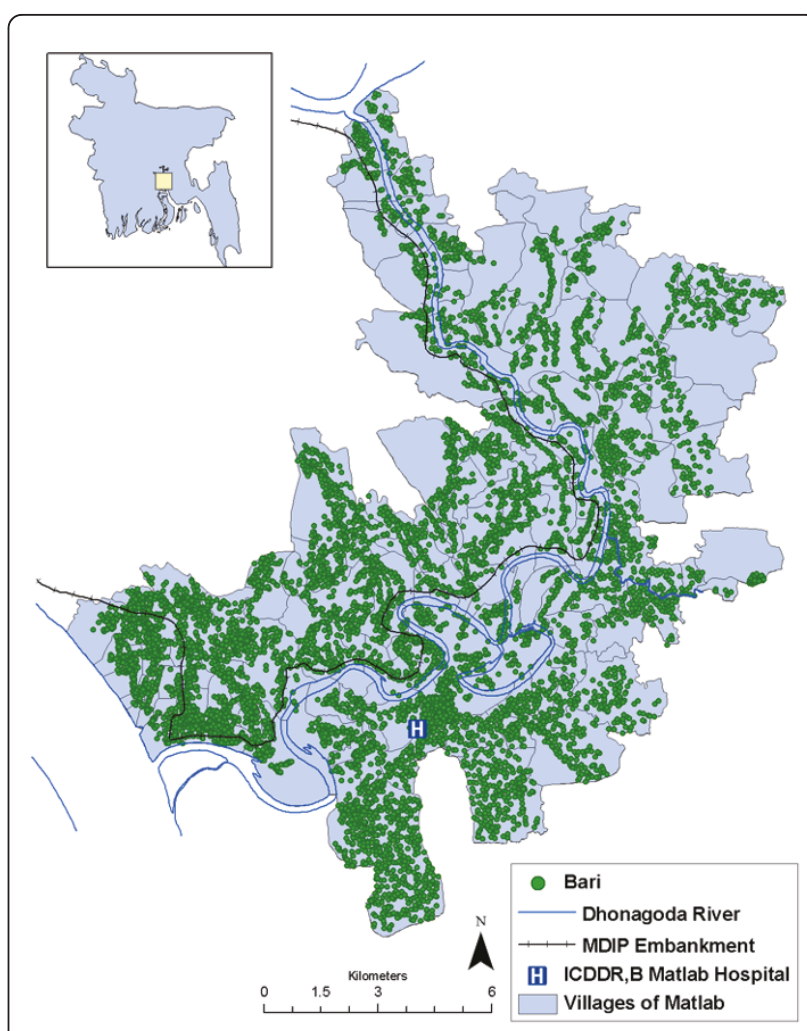

Figure 1 Location and main geographical features of the Matlab, Bangladesh study area.

coordinates of other geographic features, such as the ICDDR,B hospital. The location of features in the Matlab GIS are accurate within 10 meters [8]. The spatially-joined environmental and demographic surveillance data allows for the calculation of a variety of measures which are described in further detail below.

Multiple datasets were joined in the GIS for analysis. The first is a comprehensive global positioning systems (GPS) survey of tubewells conducted in Matlab in 2003 with hand-held receivers used for previous surveys [8]. This survey collected information on tubewell depth and arsenic level for all 10,942 tubewells in the study area. Each tubewell has a unique identification number that links it to a specific bari of ownership. For each bari we calculated the total number of available tubewells (see Figure 2) as well as an average depth statistic. The average depth statistic was chosen as an effect modifier to control for the possible confounding effects of tubewell depth on tubewell density. Approximately thirty percent of the baris in the Matlab study area do not have a privately owned tubewell; their water is drawn from public tubewells or from those belonging to neighboring baris. In such cases, their tubewell characteristics were assigned across the board as zero. This retention of baris without privately owned tubewells allows us to 


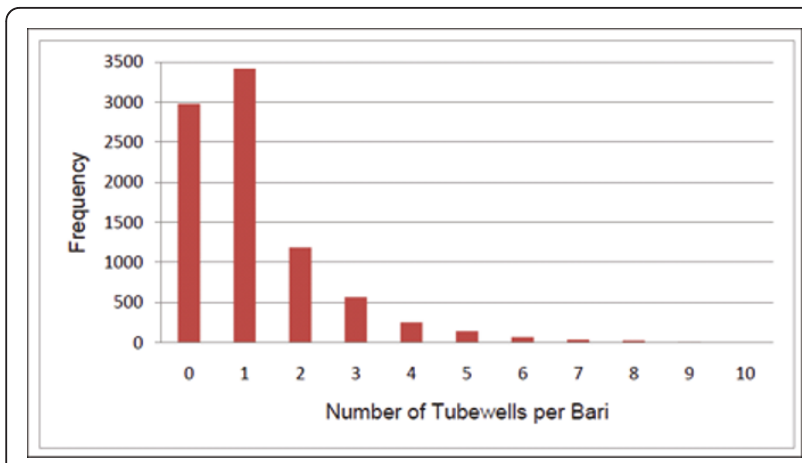

Figure 2 Distribution of bari tubewell density in Matlab, 20022004. Nineteen baris in Matlab have eleven or more tubewells each, the maximum number of tubewells per bari is 21 .

examine the effect of tubewell density on diarrheal events not only in baris with tubewells, but also among those who must either travel to a tubewell. Less convenient access to the main source of drinking water could potentially result in longer storage of drinking water within the home increasing, the likelihood of drinking water contamination if not stored properly, or increased use of surface water for convenience [7]. The distribution of mean tubewell depths in Matlab is seen in Figure 3.

All laboratory-confirmed cholera and shigellosis cases reported at the ICDDR,B hospital were aggregated by bari for the period January 1, 2002 to December 31, 2004. These years were chosen to extend one year before and one year following the tubewell GPS survey in 2003, to avoid misrepresenting tubewell density prior to the survey, and to avoid confounding by the mass installation of deep tubewells by non-governmental organizations (NGOs) in Matlab in 2005. From 2002 to 2004 there were 645 cholera cases and 527 shigellosis cases in Matlab (annual and spatial distributions are seen in Figures 4 and 5, annual incidence rates in Table 1). The total at-risk population for each bari was calculated by summing the mid-year

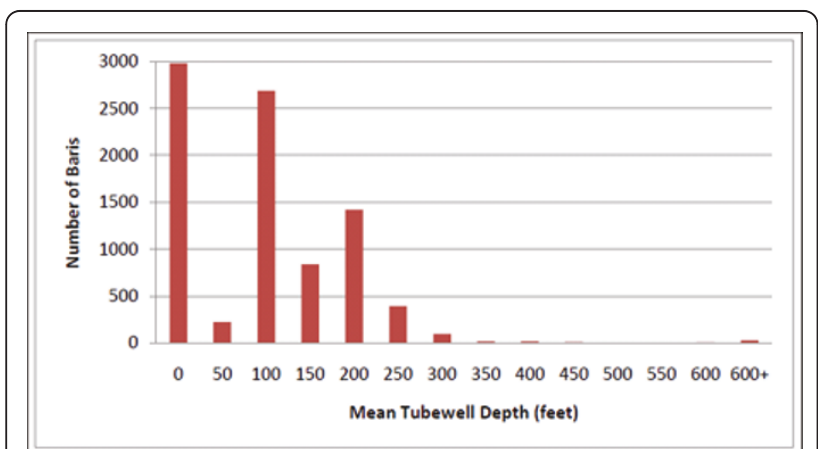

Figure 3 Distribution of mean tubewell depth in Matlab Almost three thousand baris have no private tubewell, coded as zero. The majority of tubewells in Matlab are less than 250 feet deep.

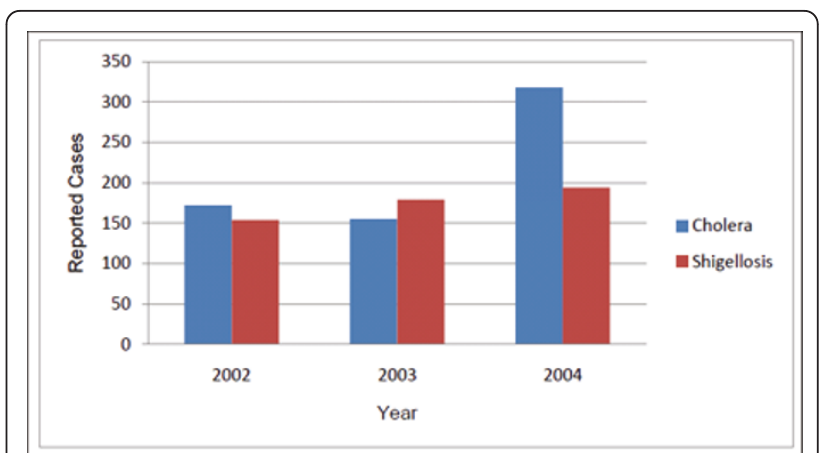

Figure 4 Annual distribution of cholera and shigellosis, 20022004, in Matlab.

background population counts for 2002 to 2004. Mid-year background populations are derived from the Matlab DSS and account for changes to a bari's population due to births, deaths and migrations. Population data gathered by ICDDR,B represents a census of all residents in any given year, and is made possible by the monthly visits of the community health workers to each bari.

The ICDDR,B Matlab hospital is not located in the geographic center of the study area, and varying distance decay effects on patients seeking treatment have been recorded [9-13]. Information regarding residents seeking treatment outside of the study area is currently unavailable. To account for this influence of distance from household to hospital on reporting of cholera and shigellosis, we calculated in the GIS the distance from each bari to the ICDDR,B hospital and controlled for it in our models. Straight-line Euclidean distance between baris and the hospital was calculated, given that residents of Matlab make use of both road and river networks to travel to the hospital. Because no specific route was known for individual baris, a universal distance metric was chosen.

\section{GWR analysis}

Traditional regression models assume that the process accounting for disease incidence is spatially stationary across the study area. This assumption, however, contradicts the goals of spatially-explicit studies whose aim is to understand where and why an outcome differs across a study area. Geographically weighted regression (GWR) models can be used to generate hypotheses about nonstationary relationships as well as pinpoint locations that should be subject to more intensive research $[14,15]$.

GWR produces two types of models: a global regression model that treats relationships as spatially stationary, and local regression models estimated at each observation point. Similar to traditional regression models, GWR estimates a model of global associations with parameter estimates, standard errors and $t$-values. Local regression models are estimated by constructing a 


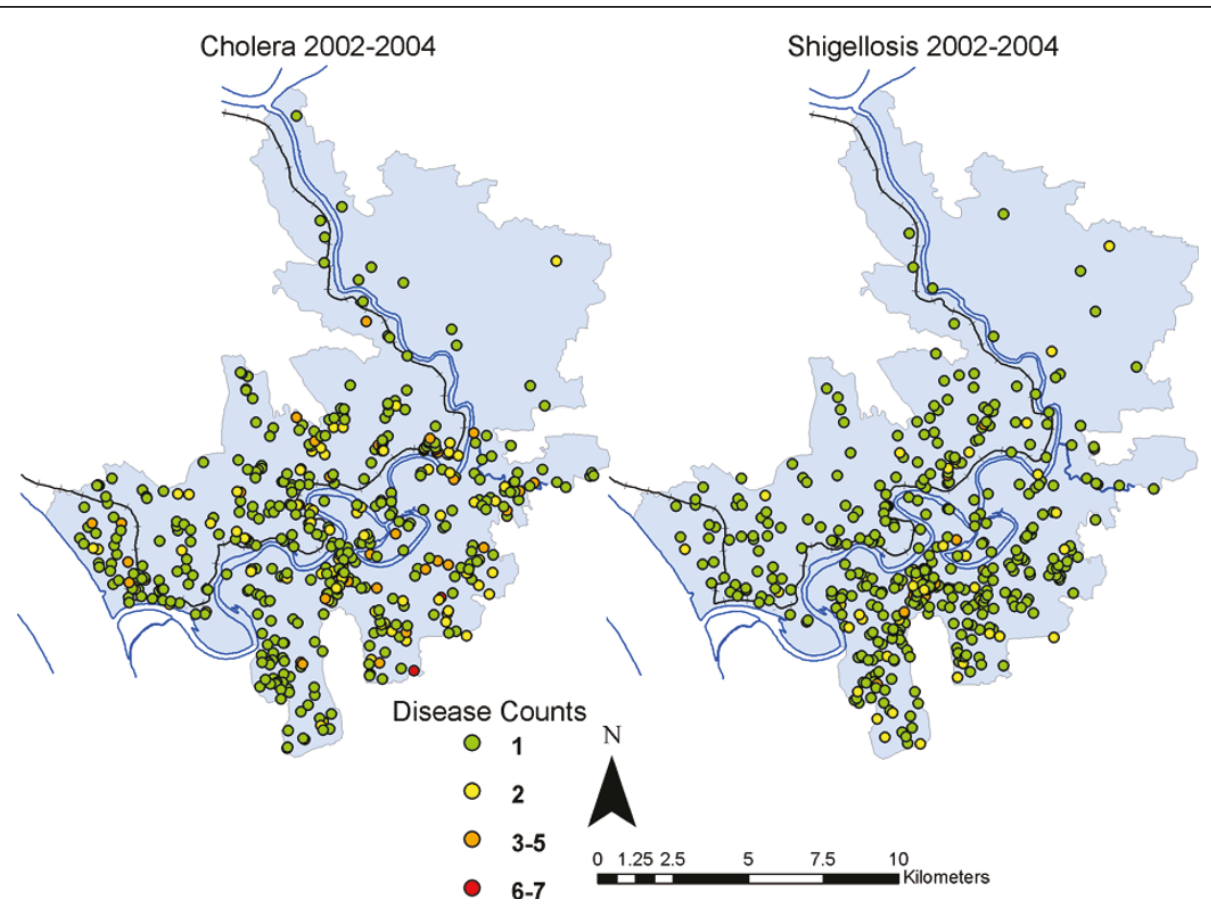

Figure 5 Spatial distribution of non-zero cholera and shigellosis counts by bari in Matlab, aggregated from 2002-2004

spatial weighting matrix and estimating a local weighted regression for each bari and its defined neighbors. Observations are weighted based on proximity to each location $i$ such that nearer points are assigned greater weight than observations further away, based upon the geographic assumption of spatial autocorrelation. A regression estimate is calculated at each observation, including $\mathrm{R}^{2}$ and other goodness-of-fit measures [15]. A Monte Carlo simulation approach is used to measure significance of local parameters. The variance of observed model parameters is compared against 100 random calibrations of the same model, providing $t$-statistics of significance. The local parameter estimates are specific to each location in both logistic and Gaussian GWR and can be displayed graphically using GIS software, indicating potential spatially-heterogeneous patterns in the relationships between diarrheal disease incidence and variables of interest.

Prior to constructing GWR models, the 8732 baris were aggregated into 666 clusters using a $25 \times 25$ meter

Table 1 Annual incidence rates (per 1000 people) for cholera and shigellosis in Matlab, Bangladesh, 2002-2004

\begin{tabular}{lccc}
\hline & \multicolumn{3}{c}{ Year } \\
\hline Disease & 2002 & 2003 & 2004 \\
\hline Cholera & 0.774367 & 0.691906 & 1.417605 \\
\hline Shigellosis & 0.693328 & 0.799039 & 0.864828 \\
\hline
\end{tabular}

grid in ArcGIS software. The grid was constructed to create a smooth, continuous surface where each cell has unique coordinates that can be analyzed using GWR. While each bari in Matlab is associated with a specific set of geographic coordinates collected at the center of the bari, the coordinates represent points rather than polygons, their dense spatial configuration made conducting GWR without construction of the $25 \times 25$ meter grid impossible. Model specifications include a logistic distribution in the absence of a negative binomial option to account for low incidence values and overdispersion, and an adaptive spatial kernel function to assign weights for each local regression estimate. The kernel bandwidth was determined by corrected Akaike Information Criterion (AICc) minimization using all data. Adaptive kernel sample size limits were between 166 and 666 bari clusters. We selected a Monte Carlo significance testing procedure for the local parameter estimates. The four variables calculated in the GIS were then analyzed against the aggregated counts of cholera and shigellosis cases reported to ICDDR,B: number of tubewells per bari, total bari population, distance to the Matlab hospital, and the mean tubewell depth per bari. Specifically, the aim of constructing geographically weighted regression models was to determine whether tubewell density effects on diarrheal events vary across space, when controlling for other covariates. Local variation would suggest that tubewell access intervention 
strategies are effective in specific areas within Matlab while a lack of spatial variation would indicate equal effects of tubewell access throughout Matlab.

\section{ZINB}

Many disease datasets contain a large number of zeroes, violating standard regression distribution assumptions and making Poisson or negative binomial regression problematic [16-19]. Zero inflated models (including both zero inflated Poisson (ZIP) and zero inflated negative binomial (ZINB)) account for this overabundance of zero counts and eliminate bias in parameter estimates, and are increasingly used in health studies $[11,20,21]$. Of the 8732 baris in Matlab from 2002 to 2004, 8278 (94.8\%) reported zero cholera events and 8308 (95.1\%) reported zero shigellosis events. A zero inflated model assumes that these zero counts are made up of both true and false zeroes. In the case of the Matlab dataset, false zeroes would arise from diseased individuals not seeking treatment at the central Matlab ICDDR,B hospital, either because of self-treatment with oral rehydration or other methods, or because medical attention was sought elsewhere in Matlab or the surrounding region. We assume that these false zeroes are greatly outnumbered by true zero counts, however, because of the Matlab ICDDR,B hospital's excellent record in treating diarrheal diseases, with free treatment options and provision of transport to sick individuals.

Zero inflated models are comprised of two components which are analyzed separately. The first component is a count model, containing all positive count observations and a proportion of zero counts that can be normally expected under either a Poisson or negative binomial distribution. The second is a binary model, where zero counts are compared to observations with any positive counts. Zero inflated models thus allow us to estimate the effects of multiple variables on both the likelihood of a bari experiencing increased magnitude of disease events (the count model), and the likelihood of a bari experiencing no cholera or shigellosis events (the binary model) $[18,22,23]$.

The $p s c l$ package in $\mathrm{R}$ was used for parameter estimation [22,24,25]. A ZINB model was selected due to the overdispersion of aggregated cholera and shigellosis counts with respect to the Poisson distribution. Using the Akaike Information Criterion (AIC) and Log-Likelihood Ratio (LLR) as indications of goodness of fit, the best set of parameters was retained.

\section{Results \\ GWR}

Results for the GWR cholera model can be seen in Tables 2 and 3. The global models represent spatially stationary Logistic regression results. Student $t$-values of \pm 1.96 indicate significance at the 0.05 level. Both total
Table 2 Parameter estimates of the cholera geographically weighted global regression model

\begin{tabular}{lccc}
\hline & \multicolumn{3}{c}{ Global Model } \\
\hline Intercept & Estimate & Std. Error & T value \\
\hline Population (02-04) & -0.425 & 0.301 & -1.413 \\
\hline Distance to hospital & 0.002 & 0.000 & $7.517^{* *}$ \\
\hline Number tubewells/bari & 0.000 & 0.000 & $-9.026^{* * *}$ \\
\hline Mean tubewell depth & -0.017 & 0.012 & -1.452 \\
\hline Significance values: ${ }^{* * * *} 0.001^{{ }^{* * *}}{ }^{0.01}{ }^{* * \prime} 0.05$ & 0.002 & 0.002 \\
\hline
\end{tabular}

bari population and average hospital distance are significant predictors of a bari cluster reporting a cholera event. The likelihood of a bari cluster reporting a cholera event increases as population increases. Baris located further from the hospital experience a distance decay effect and are less likely to report cholera. Controls for mean tubewell depth and number of tubewells per bari are not significant in the global cholera model.

All variables included in the global model were tested in the local cholera model. Decreased AIC values suggest that the local model is a better fit for the data as relationships are non-stationary. A decrease in AIC of 3 points or greater is considered evidence of an improved fit [26]. For the cholera model, the corrected AIC drops 16.76 points, suggesting that the local model is a better fit and spatial heterogeneity may exist (Table 3 ). However, using the AICc minimization for kernel sample selection resulted in an optimal bandwidth of 366 out of 666 observations. Additional bandwidths with fewer than 366 observations were tested resulting in global models with lower AIC values relative to the local models. Thus, while the drop in the AIC suggests an improved fit in the local model, convergence with more than half of the observations included in each local regression model demonstrates dominant global processes and a lack of spatial heterogeneity. Figure 6 displays the parameter estimates for the primary variable of interest in this study, number of tubewells per bari. Parameter estimates are not significant but are shown to demonstrate the limited variability among the relationship between reported cholera and tubewell density across the study area, as indicated by very small range of parameter estimates (between 0.022 and -0.059).

Table 3 Comparison of cholera global and local Logistic model diagnostics

\begin{tabular}{lll}
\hline & $\begin{array}{l}\text { Global Cholera } \\
\text { Model }\end{array}$ & $\begin{array}{l}\text { Local Cholera } \\
\text { Model }\end{array}$ \\
\hline Log Likelihood & -312.10 & -292.46 \\
\hline $\begin{array}{l}\text { Akaike Information } \\
\text { Criterion }\end{array}$ & 635.10 & 618.41 \\
\hline Corrected AIC & 636.09 & 619.33 \\
\hline
\end{tabular}




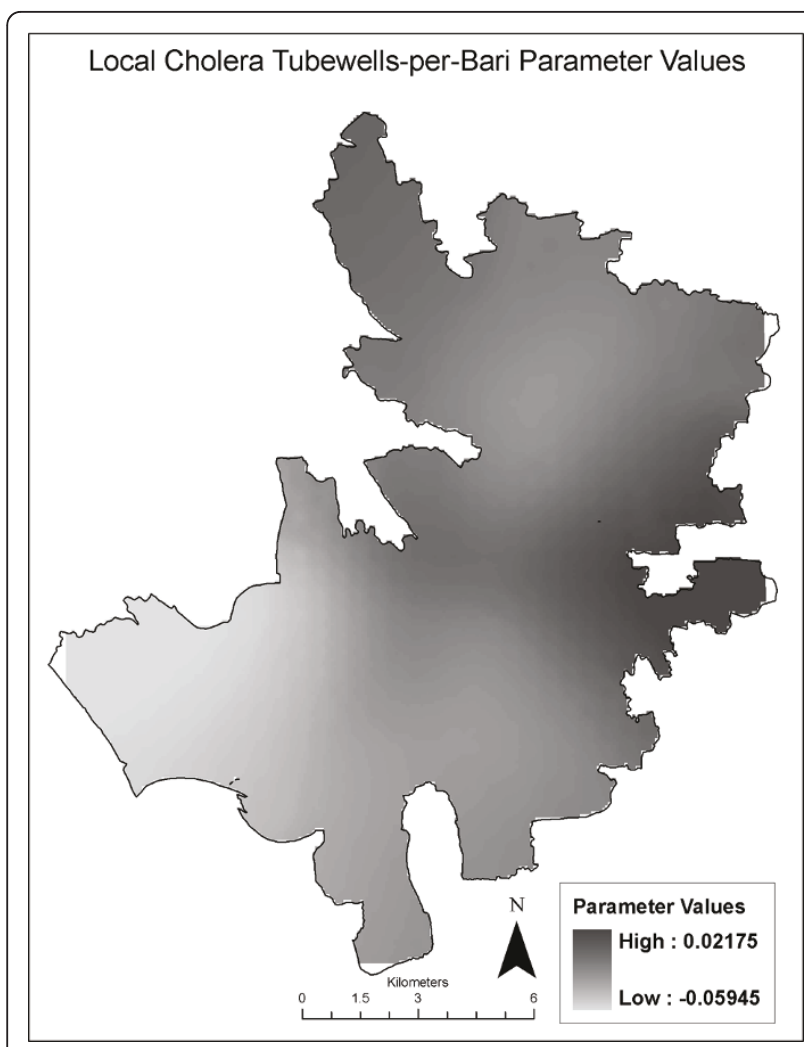

Figure 6 Local parameter estimates for the number of tubewells per bari variable, cholera model.

The global GWR results for the shigellosis model are similar to the cholera model results (Table 4). Total bari population is a significant positive predictor of reported shigellosis. Average hospital distance is a significant negative predictor of shigellosis, thus the likelihood of a bari cluster reporting a case of shigellosis between 2002 to 2004 decreases as distance to hospital increases. Mean tubewell depth and number of tubewells per bari variables are not significant predictors in the global shigellosis model.

The decrease in corrected AIC between the local and global shigellosis models is less than 3 points suggesting that the local model is not a better fit and spatial heterogeneity is not present (Table 5). Using the AICc

Table 4 Parameter estimates of the shigellosis geographically weighted global regression model

\begin{tabular}{lccc}
\hline & \multicolumn{3}{c}{ Global Model } \\
\hline Intercept & Estimate & Std. Error & T value \\
\hline Population (02-04) & 0.270 & 0.301 & 0.897 \\
\hline Distance to hospital & 0.001 & 0.000 & $5.268^{* *}$ \\
\hline Number tubewells/bari & 0.000 & 0.000 & $-10.037^{* * *}$ \\
\hline Mean tubewell depth & 0.029 & 0.239 & 0.121 \\
\hline Significance values: ${ }^{\prime * * *} 0.001^{* * *} 0.01^{* * \prime} 0.05$ & & -0.301 \\
\hline
\end{tabular}

Table 5 Comparison of shigellosis global and local Logistic model diagnostics

\begin{tabular}{lll}
\hline & $\begin{array}{l}\text { Global Shigellosis } \\
\text { Model }\end{array}$ & $\begin{array}{l}\text { Local Shigellosis } \\
\text { Model }\end{array}$ \\
\hline Log Likelihood & -314.07 & -308.48 \\
\hline $\begin{array}{l}\text { Akaike Information } \\
\text { Criterion }\end{array}$ & 638.13 & 635.58 \\
\hline Corrected AIC & 638.23 & 635.87 \\
\hline
\end{tabular}

minimization for kernel sample selection resulted in an optimal bandwidth of 571 out of 666 observations. Additional bandwidths with fewer than 571 observations were tested resulting in global models with lower AIC values relative to the local models. This is evidence of stationary relationships across the study area. Parameter estimates of local relationships between number of tubewells per bari and shigellosis are shown in Figure 7 to demonstrate the lack of spatial variability. The lack of variability in Figure 7 is the result of non-significant, uniform local parameter estimates ranging between 0.0009 and 0.0017 . GWR results for both the cholera and shigellosis models suggest limited or zero evident spatial heterogeneity in the relationships between event and predictor variables. Therefore, the use of a stationary statistical model that does not account for spatial

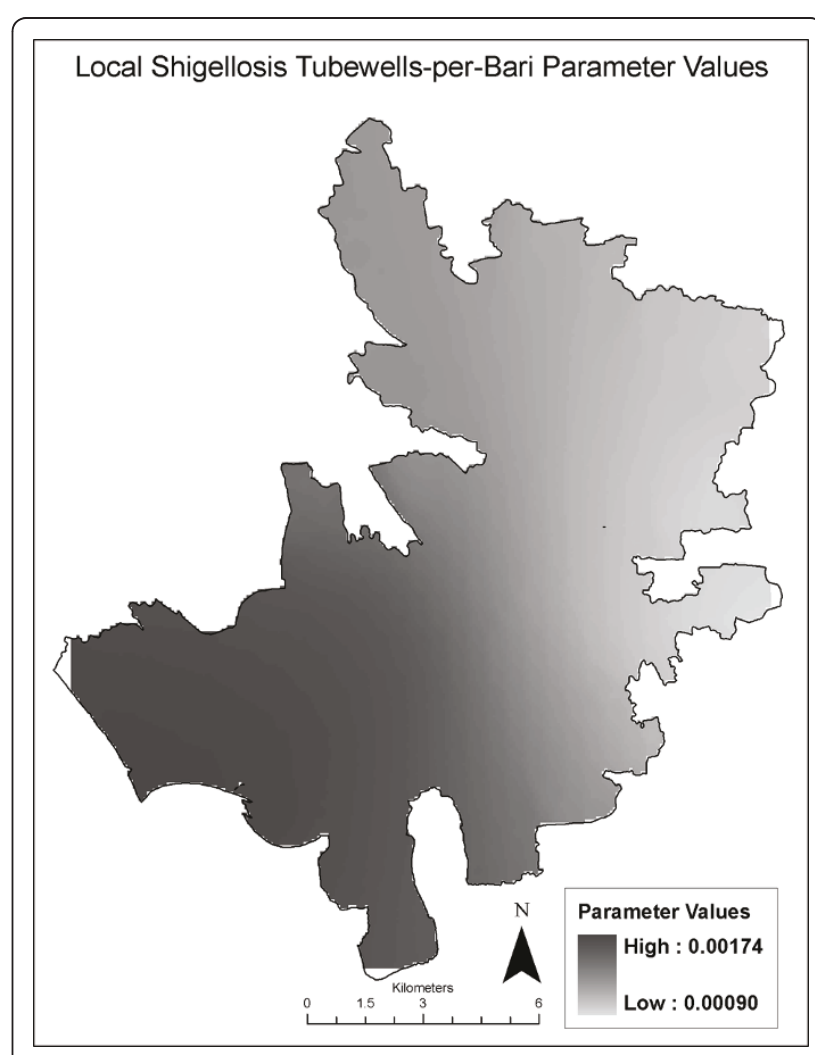

Figure 7 Local parameter estimates for the number of tubewells per bari variable, shigellosis model. 
variation in effects, but does control for low event values and the overabundance of zeros, is appropriate for analyzing these data.

\section{ZINB}

Results for the ZINB model for cholera events are presented in Table 6 and the shigellosis model results in Table 7. The expected outcome for zero inflated models is that the direction of association between variables observed in the count model will be reversed in the binary model, such that a positive association with increased counts in the count model will be matched by a negative association with zero events in the binary model.

For baris experiencing cholera in 2002 to 2004, there was a strongly significant $(\mathrm{p}<.001)$ relationship to population and hospital distance. Baris with larger populations during the time period experienced greater numbers of cholera cases. Baris that were far away from the Matlab ICDDR,B hospital experienced lower cholera counts. The number of tubewells that a bari had access to was not a statistically significant predictor of cholera counts nor was the mean tubewell depth. The binary model for cholera shows only two statistically significant relationships in the prediction of zero cholera counts: number of tubewells per bari and the background population. Increased access to tubewells, as measured by the number of tubewells per bari, significantly increases the probability of a bari reporting zero cholera events. The background population of a bari shows a statistically significant negative relationship with zero counts, such that higher bari population decreases the chances of a bari reporting no cholera. Mean tubewell depth, as in the count model, showed no statistically significant association in the binary model.

As seen in Table 7, the same predictor variables were statistically significant in the shigellosis count and binary models as the cholera models. In the count model, background bari population from 2002-2004 is a significant $(\mathrm{p}<.001)$ positive predictor of positive shigellosis counts: as population increases so does the number of shigellosis cases experienced by a bari. Distance to the
ICDDR,B hospital has the opposite effect on shigellosis case counts: baris located further from the hospital are less likely to have high numbers of reported shigellosis cases $(\mathrm{p}<.001)$. Neither the number of tubewells in a bari nor a bari's mean tubewell depth are significant predictors of shigellosis counts.

Only two variables were significant in the shigellosis binary model: the number of tubewells that a bari was able to access and the population of a bari from 20022004. As the number of tubewells in a bari increases, the likelihood of reporting zero shigellosis events increases. As population increases, the reporting of zero shigellosis events decreases at the bari level. Distance to the hospital and mean tubewell depth were not significant predictors of whether a bari experienced shigellosis during 2002 to 2004.

\section{Discussion}

Geographically weighted regression models were constructed to explore spatial heterogeneity of relationships to determine whether a stationary statistical model was appropriate for the data. Results from both the cholera and shigellosis models suggest that relationships are stationary. The influence of tubewell density on both cholera and shigellosis counts does not vary across the study area suggesting that water access interventions should apply to the whole of Matlab rather than specific target areas.

Results from both the cholera and shigellosis zero inflated models indicate that, when controlling for other population and environment covariates, the number of tubewells that a bari is able to access is an important predictor of diarrheal disease events. The models control for background bari population size, so regardless of the number of people sharing tubewells, as access to tubewell water increases at the bari level the chances of those baris reporting no cholera or shigellosis events also increases. This relationship holds true despite the presence of a mean tubewell depth variable in the model, which would control for the effect of varying tubewell depths in some baris, or the absence of tubewells completely in one third of the baris measured. It

Table 6 Parameter estimates of the cholera zero inflated negative binomial regression model

\begin{tabular}{|c|c|c|c|c|c|c|c|c|c|c|}
\hline \multirow[b]{3}{*}{ Intercept } & \multicolumn{5}{|c|}{ Count Model } & \multicolumn{5}{|c|}{ Binary Model } \\
\hline & \multirow{2}{*}{$\begin{array}{c}\text { Estimate } \\
-1.7170\end{array}$} & \multirow{2}{*}{$\begin{array}{c}\text { Std. Error } \\
0.1641\end{array}$} & \multirow{2}{*}{$\begin{array}{c}\text { z value } \\
-10.4620\end{array}$} & \multicolumn{2}{|c|}{$p$ value } & \multirow{2}{*}{$\begin{array}{c}\text { Estimate } \\
3.0713\end{array}$} & \multirow{2}{*}{$\begin{array}{c}\text { Std. Error } \\
0.4946\end{array}$} & \multirow{2}{*}{$\frac{z \text { value }}{6.2100}$} & \multicolumn{2}{|c|}{$p$ value } \\
\hline & & & & $<2 \mathrm{E}-16$ & $* * *$ & & & & $5.30 \mathrm{E}-10$ & $* * *$ \\
\hline Number tubewells/bari & -0.0001 & 0.0350 & -0.0030 & 0.9980 & & 0.6756 & 0.2192 & 3.0830 & 0.0021 & $* *$ \\
\hline Population (02-04) & 0.0047 & 0.0008 & 6.0010 & 0.0000 & $* * *$ & -0.0792 & 0.0128 & -6.1870 & $6.12 \mathrm{E}-10$ & $* * *$ \\
\hline Distance to hospital & -0.1797 & 0.0204 & -8.7900 & $<2 \mathrm{E}-16$ & $* * *$ & -0.0037 & 0.0762 & -0.0490 & 0.9611 & \\
\hline Mean tubewell depth & 0.0007 & 0.0007 & 1.0190 & 0.3080 & & 0.0005 & 0.0019 & 0.2670 & 0.7894 & \\
\hline Log (theta) & -1.2490 & 0.1252 & -9.9730 & $<2 \mathrm{E}-16$ & $* * *$ & & & & & \\
\hline
\end{tabular}

Significance values: $0^{\prime * * * \prime} 0.0001^{\prime * * \prime} 0.01^{\prime * \prime}$ 
Table 7 Parameter estimates of the shigellosis zero inflated negative binomial regression model

\begin{tabular}{|c|c|c|c|c|c|c|c|c|c|c|}
\hline \multirow[b]{3}{*}{ Intercept } & \multicolumn{5}{|c|}{ Count Model } & \multicolumn{5}{|c|}{ Binary Model } \\
\hline & \multirow{2}{*}{$\begin{array}{c}\text { Estimate } \\
-1.2265\end{array}$} & \multirow{2}{*}{$\begin{array}{c}\text { Std. Error } \\
0.2100\end{array}$} & \multirow{2}{*}{$\begin{array}{c}\mathbf{z} \text { value } \\
-5.8410\end{array}$} & \multicolumn{2}{|c|}{$\mathrm{p}$ value } & \multirow{2}{*}{$\begin{array}{c}\text { Estimate } \\
1.9978\end{array}$} & \multirow{2}{*}{$\begin{array}{c}\text { Std. Error } \\
0.3685\end{array}$} & \multirow{2}{*}{$\begin{array}{c}z \text { value } \\
5.4220\end{array}$} & \multicolumn{2}{|c|}{$p$ value } \\
\hline & & & & $5.18 \mathrm{E}-09$ & $* * *$ & & & & 5.89E-08 & $* * *$ \\
\hline Number tubewells/bari & 0.0587 & 0.0381 & 1.5390 & 0.1238 & & 0.4105 & 0.2011 & 2.0410 & 0.0412 & * \\
\hline Population (02-04) & 0.0020 & 0.0009 & 2.3180 & 0.0204 & $*$ & -0.0379 & 0.0069 & -5.5220 & $3.36 \mathrm{E}-08$ & $* * * *$ \\
\hline Distance to hospital & -0.2631 & 0.0246 & -10.7100 & $<2 \mathrm{E}-16$ & $* * *$ & 0.0662 & 0.0606 & 1.0930 & 0.2744 & \\
\hline Mean tubewell depth & 0.0010 & 0.0010 & 1.0390 & 0.2986 & & -0.0011 & 0.0028 & -0.3930 & 0.6946 & \\
\hline Log (theta) & 0.4900 & 0.4080 & 1.2010 & 0.2297 & & & & & & \\
\hline
\end{tabular}

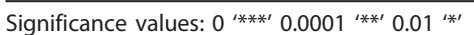

is commonly assumed that deeper tubewells draw less polluted water to the surface than shallow tubewells, which may become contaminated as surface water or other material seeps downwards towards the water table [27-29]. While we did not specifically investigate the relationship between shallow tubewells and diarrheal disease events, it was important to account for this potential interaction with the number of tubewells available to a population. Our findings indicate that when the potential for poor water quality in baris without tubewells or in baris with shallower tubewells is accounted for, the total access to tubewells in a bari is a positive predictor for the absence of diarrheal disease.

The distance between a bari and the Matlab hospital is a significant variable for both cholera and shigellosis in the count models, wherein baris further from the hospital report fewer cases of disease. We interpret these results to mean that there is a distance decay effect in Matlab, such that even in baris with disease events, fewer are reported to the hospital. This could be due to the long travel times between baris located in the further reaches of the study area, or due to residents seeking medical treatment outside the bounds of the study area. Distance to the hospital is not, however, a significant variable in either of the binary models, suggesting that it is not strongly predictive of whether a bari does or does not experience disease. The global GWR models support this assertion.

\section{Conclusions}

Our findings demonstrate the importance of access to clean water in driving diarrheal disease events in the developing world: higher densities of tubewells contribute to lower reports of cholera and shigellosis events. Increasing the amount and quality of clean water available to residents of Matlab and other regions of Bangladesh could lower not only the risk of cholera and shigellosis, but also other diarrheal diseases. This study was limited to the reporting of disease events from 2002 to 2004. Since that time, a large number of very deep tubewells (> 700 feet) have been installed in Matlab, and elsewhere in Bangladesh, by a variety of NGOs to mitigate the impacts of arsenic poisoning from shallow tubewells. Such installation could have dramatic effects on both the distribution and intensity of diarrheal disease events. As part of arsenic mitigation efforts, tubewells were labeled as safe or unsafe for drinking. Residents of baris with no safe tubewells have shown a variety of strategies, ranging from travelling to neighboring baris or public tubewells to access safe water, switching usage to only one tubewell in a bari, or ignoring the warnings and still using arsenic infected tubewells [30]. Our future work will examine the durability of these results in the face of changing water access in Matlab, driven both by individual arsenic mitigation efforts (switching tubewells) and by the large-scale installation of even deeper tubewells by the Bangladeshi government and NGOs.

\section{Acknowledgements}

This study was conducted with the support of ICDDR,B and its donors which provide unrestricted support to ICDDR,B for its operations and research. Current donors providing unrestricted support include: the Government of the People's Republic of Bangladesh (GoB), the Canadian International Development Agency (CIDA), Embassy of the Kingdom of the Netherlands (EKN), the Swedish International Development Cooperative Agency (SIDA), and the Department for International Development, UK (DFID). We gratefully acknowledge these donors for their support and commitment to ICDR,B's research efforts. This study was also supported by the National Oceanic and Atmospheric Administration Oceans and Human Health Program and the National Science Foundation (BCS-0924479) and the IGERT program at the Carolina Population Center).

\section{Author details}

${ }^{1}$ Department of Geography, University of lowa, lowa City, IA, USA

${ }^{2}$ Department of Geography \& Carolina Population Center, University of North Carolina-Chapel Hill, Chapel Hill, NC, USA. International Center for Diarrheal Disease Research, Bangladesh, Dhaka, Bangladesh.

\section{Authors' contributions}

$M C, V E$ and ME designed the study, and with JM carried out the statistical analysis and drafted the manuscript. MY and PKS oversaw data design and collected the dataset, and helped revise the manuscript. SG and JW participated in the design of the study and helped to draft the manuscript. All authors read and approved the final manuscript.

\section{Competing interests}

The authors declare that they have no competing interests.

Received: 6 January 2011 Accepted: 15 June 2011

Published: 15 June 2011 


\section{References}

1. Islam MS, Siddika A, Khan MNH, Goldar MM, Sadique MA, Kabir A, Huq A, Colwell RR: Microbiological analysis of tube-well water in a rural area of Bangladesh. Appl Environ Microbiol 2001, 67(7):3328.

2. Hoque B, Juncker T, Sack R, Ali M, Aziz K: Sustainability of a water, sanitation and hygiene education project in rural Bangladesh: a 5-year follow-up. Bull World Health Organ 1996, 74(4):431.

3. Tumwine JK, Thompson J, Katua-Katua M, Mujwajuzi M, Johnstone N, Porras I: Diarrhoea and effects of different water sources, sanitation and hygiene behaviour in East Africa. Trop Med Int Health 2002, 7(9):750-756.

4. Esrey SA: Water, waste, and well-being: a multicountry study. Am J Epidemiol 1996, 143(6):608

5. Aziz K, Hoque BA, Hasan KZ, Patwary M, Huttly SRA, Rahaman MM, Feachem R: Reduction in diarrhoeal diseases in children in rural Bangladesh by environmental and behavioural modifications* 1 . Trans $R$ Soc Trop Med Hyg 1990, 84(3):433-438.

6. Montgomery MA, Elimelech M: Water and sanitation in developing countries: including health in the equation. Environ Sci Technol 2007. 41(1):17-24.

7. Hoque BA, Hallman K, Levy J, Bouis H, Ali N, Khan F, Khanam S, Kabir M, Hossain S, Shah Alam M: Rural drinking water at supply and household levels: Quality and management. Int J Hyg Environ Health 2006, 209(5):451-460.

8. Ali M, Emch M, Ashley C, Streatfield PK: Implementation of a medical geographic information system: concepts and uses. J Health Popul Nutr 2001, 19(2):100-110.

9. Ali M, Emch M, Donnay JP, Yunus M, Sack RB: The spatial epidemiology of cholera in an endemic area of Bangladesh. Soc Sci Med 2002, 55(6):1015-1024.

10. Ali M, Emch M, Donnay JP, Yunus M, Sack RB: Identifying environmental risk factors for endemic cholera: a raster GIS approach. Health Place 2002, 8(3):201-210.

11. Carrel M, Voss P, Streatfield PK, Yunus M, Emch M: Protection from annual flooding is correlated with increased cholera prevalence in Bangladesh: a zero-inflated regression analysis. Environ Health 2010, 9:13.

12. Emch M: Diarrheal disease risk in Matlab, Bangladesh. Soc Sci Med 1999, 49(4):519-530.

13. Emch M, Ali M, Yunus M: Risk areas and neighborhood-level risk factors for Shigella dysenteriae 1 and Shigella flexneri. Health Place 2008, 14(1):96-105.

14. Brunsdon C, Fotheringham S, Charlton M: Geographically Weighted Regression-Modelling Spatial Non-Stationarity. The Statistician 1998, 47(3):431-443.

15. Fotheringham AS, Charlton ME, Brunsdon C: Geographically weighted regression: a natural evolution of the expansion method for spatial data analysis. Environ Plann A 1998, 30:1905-1927.

16. Cameron AC, Trivedi PK: Econometric models based on count data: comparisons and applications of some estimators and tests. J App/ Econometrics 1986, 1:29-53.

17. Heilbron DC: Zero-altered and other regression models for count data with added zeros. Biometrical Journal 1994, 36(5):531-547.

18. Lambert D: Zero-inflated Poisson regression, with an application to defects in manufacturing. Technometrics 1992, 34:1-14.

19. Ridout $M$, Demetrio CGB, Hinde J: Models for count data with many zeros. Proceedings of the Proceedings of the XIXth International Biometric Conference 1998, 19:179-192.

20. Böhning D, Dietz E, Schlattmann P, Mendonça L, Kirchner U: The zeroinflated Poisson model and the decayed, missing and filled teeth index in dental epidemiology. Journal of the Royal Statistical Society: Series A (Statistics in Society) 1999, 162(2):195-209.

21. Cheung YB: Zero-inflated models for regression analysis of count data: a study of growth and development. Stat Med 2002, 21(10):1461-1469.

22. Zeileis A, Kleiber C, Jackman S: Regression Models for Count Data in R. Journal of Statistical Software 2008, 27(8):1-25.

23. Cameron AC, Trivedi PK: Regression analysis of count data. Cambridge; New York: Cambridge University Press; 1998.

24. R Development Core Team: R: A language and environment for statistical computing. 2011.

25. Jackman S: pscl: Classes and methods for R developed in the Political Science Computational Laboratory, Stanford University. 2008, R package version 1.02 .
26. Fotheringham AS, Brunsdon C, Charlton M: Geographically weighted regression: the analysis of spatially varying relationships. Chichester: Wiley; 2002

27. Howard G, Pedley S, Barrett M, Nalubega M, Johal K: Risk factors contributing to microbiological contamination of shallow groundwater in Kampala, Uganda. Water Res 2003, 37(14):3421-3429.

28. Morris BL, Lawrence ARL, Chilton P, Adams B, Calow RC, Klinck BA: Groundwater and its susceptibility to degradation: a global assessment of the problem and options for management. 2003, 03-3, Early warning and assessment report series, RS.

29. Zingoni E, Love D, Magadza C, Moyce W, Musiwa K: Effects of a semiformal urban settlement on groundwater quality:: Epworth (Zimbabwe): Case study and groundwater quality zoning. Physics and Chemistry of the Earth, Parts A/B/C 2005, 30(11-16):680-688.

30. Madajewicz M, Pfaff A, Van Geen A, Graziano J, Hussein I, Momotaj H, Sylvi R, Ahsan $\mathrm{H}$ : Can information alone change behavior? Response to arsenic contamination of groundwater in Bangladesh. J Dev Econ 2007, 84(2):731-754.

doi:10.1186/1476-072X-10-41

Cite this article as: Carrel et al:: Diarrheal disease risk in rural Bangladesh decreases as tubewell density increases: a zero-inflated and geographically weighted analysis. International Journal of Health Geographics 2011 10:41

\section{Submit your next manuscript to BioMed Central and take full advantage of:}

- Convenient online submission

- Thorough peer review

- No space constraints or color figure charges

- Immediate publication on acceptance

- Inclusion in PubMed, CAS, Scopus and Google Scholar

- Research which is freely available for redistribution

Submit your manuscript at www.biomedcentral.com/submit
C BioMed Central 